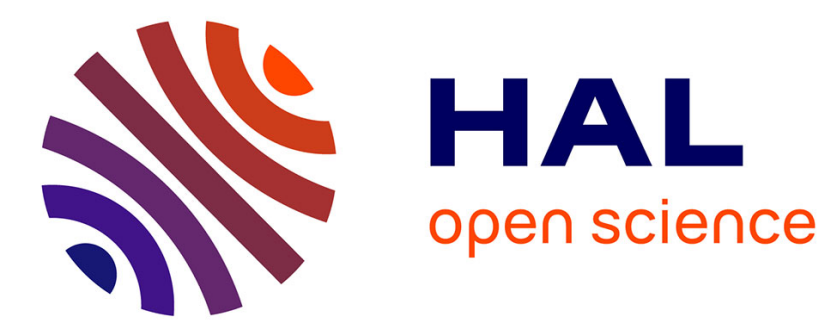

\title{
Multichannel access for bandwidth improvement in IEEE 802.15.4 wireless sensor networks
}

\author{
Stéphane Lohier, Abderrezak Rachedi, Ismail Salhi, Erwan Livolant
}

\section{To cite this version:}

Stéphane Lohier, Abderrezak Rachedi, Ismail Salhi, Erwan Livolant. Multichannel access for bandwidth improvement in IEEE 802.15.4 wireless sensor networks. IFIP/IEEE Wireless Days 2011

(IEEE WD'2011), Oct 2011, Niagara Falls, Ontario, Canada. pp.6, 10.1109/WD.2011.6098151. hal-00680871

\section{HAL Id: hal-00680871 \\ https://hal.science/hal-00680871}

Submitted on 2 May 2012

HAL is a multi-disciplinary open access archive for the deposit and dissemination of scientific research documents, whether they are published or not. The documents may come from teaching and research institutions in France or abroad, or from public or private research centers.
L'archive ouverte pluridisciplinaire HAL, est destinée au dépôt et à la diffusion de documents scientifiques de niveau recherche, publiés ou non, émanant des établissements d'enseignement et de recherche français ou étrangers, des laboratoires publics ou privés. 


\title{
Multichannel Access for Bandwidth Improvement in IEEE 802.15.4 Wireless Sensor Networks
}

\author{
Stéphane Lohier, Abderrezak Rachedi, Ismail Salhi, Erwan Livolant \\ Computer Science Laboratory (LIGM) \\ University of Paris-Est Marne-la-Vallée (UPEMLV) \\ Champs sur Marne, France \\ \{lohier, rachedi, salhi, livolant $\} @$ univ-mlv.fr
}

\begin{abstract}
In this paper, we propose a new multichannel allocation protocol for ZigBee/IEEE 802.15.4 networks. The main goal is to improve the global throughput which is basically insufficient to satisfy high bandwidth requirements for applications like monitoring or traffic control. The solution is based on the availability of multiple channels on current lowcost, low-energy radio transceivers, such as $\mathrm{CC2420}$, which can be easily tuned dynamically to different frequencies. This possibility can be exploited to increase the number of simultaneous transmissions on adjacent links. The allocation of the different channels is centralized and distributed by the coordinator thanks to a function designed to compute the channel offset between two successive children routers. In the nodes, the switching process between the transmission and the reception channels is triggered starting from the PHY primitive available on the transceiver. The evaluation shows that the proposed protocol improves the global throughput by a factor between 2 and 5, depending on the scenario, compared to the single-channel solution or a random channel allocation.
\end{abstract}

Keywords: Multichannel, Wireless Sensor Networks, IEEE 802.15.4

\section{INTRODUCTION}

The bandwidth available in Wireless Sensor Networks (WSN) is generally insufficient to satisfy greedy applications such as low/medium resolution video monitoring. For instance, the theoretical throughput in prevalent WSN standards such as ZigBee or 6LowPan is upper-bounded to250kbps. As wireless sensor networking evolves toward multimedia sensing, it becomes necessary to find new mechanisms to transmit more efficiently audio or video flows.

This study considers realistic application scenarios with high bandwidth requirements and takes into account the characteristics of existing sensing technologies. These scenarios are typically part of the paradigm that is Multimedia Sensor Networking [5] wherein sensing devices are more powerful and embedded with CMOS cameras and audio sensors. In such networks, nodes are deployed in a specific area in order to provide various services like traffic congestion control, video monitoring, object tracking and recognition, etc. In this work, we consider an IEEE 802.15.4 wireless sensor network with a hierarchical topology: coordinator, router and terminal divided in several levels of clusters. The transmissions use the 802.15.4 non-beaconed mode which allows the best bandwidth utilization [15].
In addition, we are interested here only on single interface solutions. Multiple interface schemes are not adapted to ZigBee devices because of their energy consumption limitations. In WSNs standards, much smaller packet sizes are used (e.g. 100 bytes for IEEE 802.15.4) compared to those in wireless ad hoc networks (e.g. 1500 bytes in IEEE 802.11). The solutions with control packets like RTS/CTS in IEEE 802.11 will not be adopted as they can lead to an important overhead.

Current low-cost, low-energy radio transceivers, such as CC2420 [3] can be tuned dynamically to different frequencies allowing PHY protocols to operate on different radio channels. The availability of multiple channels on the wireless medium can be exploited to increase the number of simultaneous transmissions between different pairs of transmitter/receiver. Thus, the global network throughput can potentially be increased. However, no channel switching protocol is proposed in IEEE 802.15.4 standard. That is why, in this paper, we propose a new protocol for the channels assignment named MASN (Multichannel Access for Sensor Networks), based on an offset function comparable to the one used in the hierarchical ZigBee addressing. Channels on the different links are successively allocated by the coordinator after the association process, starting from the first children routers and toward the end terminals. The proposed protocol ensures that two neighboring links will not be affected by the same channel. In addition, the protocol introduces a switching process between transmission and reception channels. This process is triggered starting from the PHY primitive available into the transceiver.

Insofar as the protocol does not modify the medium access method but only adds channel allocation and channel switching process in the nodes, it can be easily implemented in IEEE 802.15.4 device. The MASN protocol is thus designed in accordance with the 802.15.4/ZigBee networks standard which is not the case of many proposed solutions in literature.

The rest of the paper is divided into five sections. In section 2, we sum up the existing works dealing with multichannel access in wireless networks. We present our MASN protocol in section 3. Section 4 is dedicated to the performance evaluation of the proposed protocol. The fifth section concludes our paper and presents the future works. 


\section{RELATED WORKS}

In many studies designed for general wireless networks $[6,7,8]$, transmission are divided into two period: a control period with a common channel to exchange information between all nodes about free channels or to schedule channels available for future data exchanges (this period is often called ATIM window for Ad Hoc Traffic Indication Messages window); a period for simultaneous data transmissions between several pair of sender/receiver in a channel selected during the control period. These protocols are not adapted to an 802.15.4 context as all nodes' interfaces have to listen to the common channel during frequent and periodic negotiations which can be very greedy in terms of energy consumption.

The SSCH protocol [9] proposes a different approach where each node computes a channel schedule which contains the list of channels that a node plans to switch to in subsequent slots (the time spent on a single channel). To ensure that communications with neighbors are possible in some slots, each node has to maintain a list of the channel schedules for all other nodes it is aware of. The principal drawbacks of this approach in our WSN context is the high control traffic used by all nodes to periodically broadcast the channel schedule and the necessity of synchronization between nodes.

In order to consider more specifically the WSNs constraints, other works are proposed. As for MMAC [7], the TFMAC protocol [10] divides time into a fixed number of slots and allows each node to use different frequencies within different time slots to send data packets to its neighbors. The authors propose to create a TDMA frame with contention period and contention-free period which already exist in the 802.15.4 standard with the superframe in the beaconed mode. No indication is given on how to synchronize the TDMA frame between the nodes (in IEEE 802.15.4, the coordinator synchronizes the superframe on a common channel).

The MMSN protocol presented in [11] is also especially designed for WSNs and consists of two aspects: frequency assignment and media access with a specific design, different from the classical CSMA. The global throughput is significantly improved with MMSN, but the comparisons are carried out with a theoretical CSMA MAC layer and not with a real MAC IEEE 802.15.4 layer. Moreover, selected topologies are not hierarchical and the traffic pattern is many-to-many and not many-to-one like in usual WSNs or more specifically in MWSNs.

Soroush et al. proposed a hybrid TDMA/FDMA MAC protocol called HYMAC [12] in which data gathered by sensor nodes are delivered to a base station (many-to-one traffic). The transmissions take place in a fixed-length TDMA cycle divided into several fixed time slots. The base station assigns an appropriate frequency (FDMA) and a specific time slot(s) to each node (TDMA) by running a scheduling algorithm. The simulation results show that HYMAC outperforms MMSN for the number of potential conflicts but these performances are obtained by adding time slots when the number of frequency is insufficient which increases the risk of interferences. Consequently, the MAC layer is specific and not directly compatible with the IEEE 802.15.4 MAC layer or another CSMA/CA MAC layer.
In [13], $\mathrm{Wu}$ et al. propose TMCP, a tree-based multichannel protocol. The main idea is to assign channels to different subtrees instead of nodes in order to avoid interference between nodes of different subtrees (inter-tree) without the need for time synchronization. A comparison shows that the performances of TMCP and MMSN [11] are very close. The main drawback of TMCP protocol, according to our selected context, is the lack of coordination between the tree-based channel assignment scheme and the hierarchical association process already existing in ZigBee networks.

MCMAC [14] is another protocol designed for multichannel access where the sensor network is divided into clusters like in a hierarchical ZigBee network. The proposed protocol increases the network energy efficiency and the data throughput thanks to a time slotted access in a synchronous beaconed mode. Even if the average throughput is better than that obtained with a mono-channel access, the performances of the beaconed mode remain globally insufficient [15] for multimedia flows.

\section{MASN PROTOCOL}

\section{A. Context and goals}

As mentioned above, this study takes into consideration the technical characteristics of existing WSNs and relates to sensor networks with high bandwidth demand in which data are destined to a unique sink. Such context imposes some assumptions for the network and its components:

- $\quad$ static nodes limited to 50 and distributed on a surface lower than 100x100m (current deployments of ZigBee networks in home automation or monitoring contexts are generally composed of less than 50 nodes);

- hierarchical clustered topology with a single sink;

- ZigBee/IEEE 802.15.4 technology for routing, MAC and PHY layers;

- hierarchical ZigBee routing for many-to-one transmissions (different from a reactive ad hoc routing for many-to-many communications which is the case of many studies on the subject [6-11]);

- a single radio interface by node (except for the ZigBee coordinator which serves as sink);

- the IEEE 802.15.4 technology proposes a total of 16 non-overlapping channels the $2,4 \mathrm{GHz}$ band; one control channel is dedicated to the association process (channel 11) and 15 channels are used for the data transmissions (channel 12 to 26);

- the change of channel respects the switching time of the radio transceiver (typically $200 \mu$ s for the CC2420);

- the CSMA/CA access method in the non-beaconed mode is chosen as it provides the best throughput [15].

Furthermore, we chose neither to use a temporal window on a common control channel to negotiate periodically a data channel between receivers and transmitters [6-8] nor control MAC frames (RTS/CTS), as these solutions consume time and energy (cf. section II). 
Finally, the proposed protocol must be able to be easily integrated on usual sensors equipped with a CC2420 transceiver (for example TelosB, MicaZ and Imote2 provided by MEMSIC, formerly Crossbow, [4]) without important modification of the MAC layer: the medium access method is not modified (case of the algorithms with a control window).

\section{B. MASN Overview}

\section{1) Channel allocation}

The algorithm proposed for the channel allocation is inspired by the hierarchical address allocation process used in the ZigBee networks. In this process, the Cskip function is used to compute the address offset between two successive children routers for a given network depth, $d$ :

$$
\operatorname{Cskip}(d)=\left\{\begin{array}{c}
1+C m \cdot(L m-d-1), \text { if } R m=1 \\
\frac{1+C m-R m-C m \cdot R m^{L m-d-1}}{1-R m}, \text { otherwise }
\end{array}\right.
$$

$\mathrm{Lm}$ being the maximum depth in the network, $\mathrm{Cm}$ the maximum number of children a parent may have and $R m$ the maximum number of routers a parent may have as children.

Then, network addresses are assigned to end-devices in a sequential manner with the $n^{\text {th }}$ address, $A_{n}$ given by the following equation:

$$
A_{n}=A_{\text {parent }}+\operatorname{Cskip}(d) \cdot R m+n
$$

Where $1 \leq n \leq(\mathrm{Cm}-\mathrm{Rm})$ and $A_{\text {parent }}$ represents the address of the parent.

In ZigBee specifications [1], three roles are possible for a node: ZC is the Coordinator, ZR is a Router (these two roles corresponding to an 802.15.4 MAC Full Function Device) and ZED is an End Device (Reduced Function Device).

In our algorithm, like in HYMAC [12], the coordinator distributes the channels after the node-association process. The channel allocation is related to the ZigBee network parameters $\mathrm{Lm}, \mathrm{Cm}, \mathrm{Rm}$. Insofar as the values of these three parameters characterize the size of the network, the frequencies allocation is also directly limited by these values.

Each node uses one channel to send data $(C H S)$ towards its single parent. In our solution, the number of reception channels $(\mathrm{CHR})$ for a $\mathrm{ZR}$ is also limited to one. This limitation avoids a commutation algorithm between several $C H R$ based on TDMA multiplexing or on advertising frames sent on the common channel. Indeed, these two solutions would involve additional delays and would be difficult to integrate in a standard device. Moreover, the first solution (TDMA) would be contrary to the initial choice which is to use a CSMA mode in order to optimize the global throughput. The number of possible channels $\left(n_{C H S}\right.$ and $\left.n_{C H R}\right)$ for the different roles is summarized in the example depicted in figure 1.

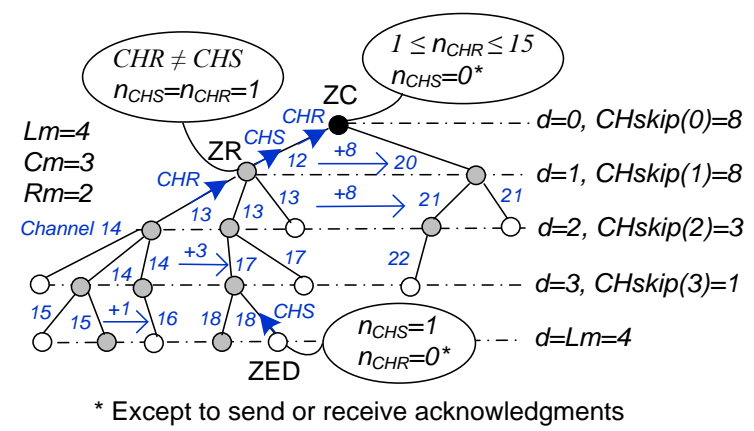

Figure 1. Example of Channel Allocation

A function comparable to $\operatorname{Cskip}(d)$, named $C H s k i p(d)$ is used to compute the channel offset between two successive children ZR. Insofar as the allocation of the $C H R$ is necessary only for the ZR (as indicated previously, the ZC can have several radio interfaces with one channel per interface), $C H \operatorname{skip}(d)$ depends on $\mathrm{Rm}$ and not on $\mathrm{Cm}$. For a given value of $L m$ and $R m$, for example $L m=4$ and $R m=2$ as in figure 1, CHskip(d) can be computed as follows:

$$
\begin{aligned}
& \operatorname{CHskip}(2)=1+R_{m}=3 \\
& \operatorname{CHskip}(1)=\operatorname{CH} \operatorname{Hsip}(0)=1+1+\left(1+R_{m}\right) R_{m}=8
\end{aligned}
$$

Finally, we can generalize the $\operatorname{CHskip}(d)$ expression:

$$
\text { CHskip }(d)=\left\{\begin{array}{l}
1+\sum_{n=0}^{L m-2} R m^{n}, \text { if } d \leq 1 \\
\sum_{n=0}^{L m-d-1} R m^{n}, \text { otherwise }
\end{array}\right.
$$

Then, the CHR values are assigned to the $\mathrm{ZR}$ in a sequential manner with the $n^{\text {th }}$ channel, $C H R_{n}$, given by the following equation:

$$
C H R_{n}=\left\{\begin{array}{c}
{[12+\operatorname{CHskip}(d) .(n-1)] \bmod 15, \text { if } d=0} \\
{\left[C H R_{\text {parent }}+1\right] \bmod 15, \text { if } d=1} \\
{\left[\text { CHR } R_{\text {parent }}+1+\operatorname{CHskip}(d) .(n-1)\right] \bmod 15, \text { otherwise }}
\end{array}\right.
$$

This allocation is illustrated in figure 1 with the different values of CHskip(d) at different depths and with the successive allocated channels. Let us note that the solution combines a channel assignation not only to different subtrees, like in TMCP [13], but also to the different hops in the same subtree. It is demonstrated in [17] that a multihop multichannel allocation scheme is efficient in an 802.15.4 network.

With these equations, the channel distribution does not directly guarantee, like in MMSN [11], that two neighboring links will have a different $C H R$ (the offset can be equal to 15 which is the number of possible channels) but the probability is weak with 15 channels and a hierarchical distribution ( 2 routers associated to the same parent will always have two distinct channels). However, to avoid this case, the algorithm attributes $C H R=n+1$ instead of $C H R=n$ for a ZR having a neighboring router of the same parent and with $C H R=n$.

In addition, as for the hierarchical addressing process, the centralized channels allocation can be reorganized if the 
network is modified because of the transition to idle state, the stop or the displacement of a node.

Finally, the algorithm validated on a ZigBee network with one $\mathrm{ZC}$ can be extended to the other ZigBee networks considered then as clusters with ZC acting as Cluster Head which distributes channels on the links.

\section{2) Channel switching}

CHR is selected by default on a ZR. On reception of a PHY primitive PD_DATA_REQUEST() indicating a data frame transmission request, $\mathrm{ZR}$ switches to CHS (if it is not receiving). The change of channel is effective after the device switching time (figure 2). ZR returns to CHR when the MAC acknowledgment is received, after another switching time. A timeout is triggered for all the data transmissions, at expiration of this timeout and in the absence of a MAC acknowledgment, the node automatically returns to CHR.

As in any ZigBee network, it is necessary to be able to reassociate a node which becomes orphan because of a link failure due to the extinction of the parent node or to interferences in the vicinity. The control channel is thus periodically switched following a command sent by the coordinator to all the nodes.

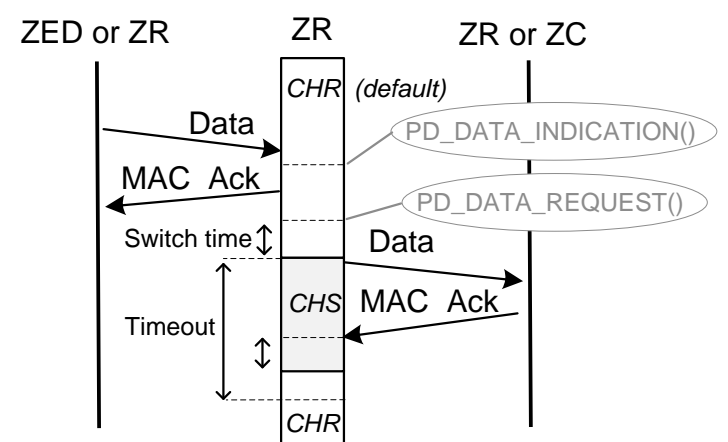

Figure 2. Channel switching Timing

\section{PERFORMANCE EVALUATION}

\section{A. Simulation scenario}

The parameters used in NS2 simulator [16] are adapted from those of the CC2420 transceiver, in particular for the channel switching time $(200 \mu \mathrm{s})$. They are summarized in table 1. The selected scenario is described in figure 3 , it corresponds to a hierarchical clustered topology with a maximum depth of 4 hops $(L m=4)$ and a maximum of 3 children by router including 2 children routers $(\mathrm{Cm}=3$ and $\mathrm{Rm}=2)$. The distances between nodes are chosen so that each node has 1 or 2 hops neighbors. The CBR sources are distributed in the ZED of the different clusters with a maximum of 25 sources and a maximum CBR frequency of 30 packets/s; these values are selected to reach the saturation of the network.

Four solutions are compared: mono-channel; our MASN protocol; a random allocation of the 15 data channels (named Random) and an allocation of a different channel for each subtree of the network (named Subtree) comparable with the one used in TMCP [13] but without the tree building process which is carried out in our solution by the ZigBee protocol. For the two last solutions, as for MASN, only one reception channel is allocated for each child router.

TABLE 1: SimUlation PARAMETERS

\begin{tabular}{|l|l|}
\hline Physical Channel & $802.15 .4-2.4 \mathrm{GHz}$ \\
\hline Data Rate & $250 \mathrm{kbps}$ \\
\hline Reception range (Capture Threshold) & $15 \mathrm{~m}$ \\
\hline Detection range (Carrier Sense Threshold) & $30 \mathrm{~m}$ \\
\hline Maximum surface & $100 \times 100 \mathrm{~m}$ \\
\hline Number of nodes & 46 \\
\hline Propagation Model & TwoRayGround \\
\hline CBR packet size & $97 \mathrm{Bytes}$ \\
\hline Routing Protocol & ZBR \\
\hline Simulation Time & $60 \mathrm{~s}$ \\
\hline
\end{tabular}

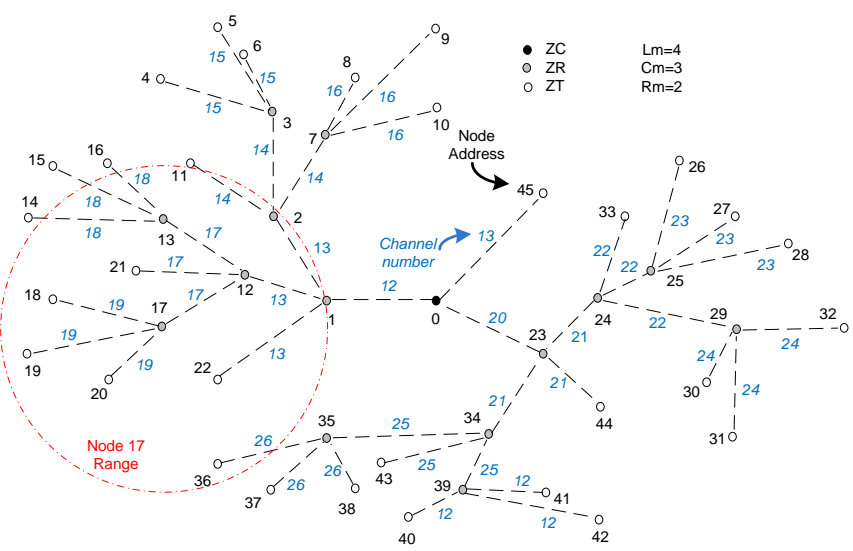

Figure 3. Simulated Network

\section{B. Simulation Results}

Figure 4 shows the evolution of the network global throughput according to the CBR sources frequency, when almost $50 \%$ of the sources (12/25) are activated. For the monochannel solution, the throughput is always lower than $60 \mathrm{kbps}$ and decreases for frequencies greater than 8 packets per second. The numerous contentions on the data common channel induce important latencies related to the CSMA/CA algorithm. The global throughput obtained with MASN protocol increases linearly to reach a value close to $190 \mathrm{kbps}$ at a frequency of 30pkts/s, this threshold is not the maximum but beyond, the delivered packets ratio becomes lower than $50 \%$. This ratio is represented in figure 5 for the four solutions. The saturation in the mono-channel is confirmed by this measurement: delivered packets ratio is lower than $80 \%$ for frequencies greater than $8 \mathrm{pkts} / \mathrm{s}$. This ratio remains higher than $90 \%$ up to a frequency of $10 \mathrm{pkts} / \mathrm{s}$ in the multichannel solution, which shows the weak number of contentions and thus the efficiency of the solution.

The random allocation gives intermediate performances insofar as it does not avoid identical channels on adjacent or neighboring links, which can involve conflicts. For the Subtree 
solution, the performances are close to those obtained with MASN for weak traffics (CBR frequencies lower than $15 \mathrm{pkts} / \mathrm{s})$. For higher traffics, the throughput and the delivered packets ratio decrease because of the numerous conflicts on the subtrees sharing the same channel.

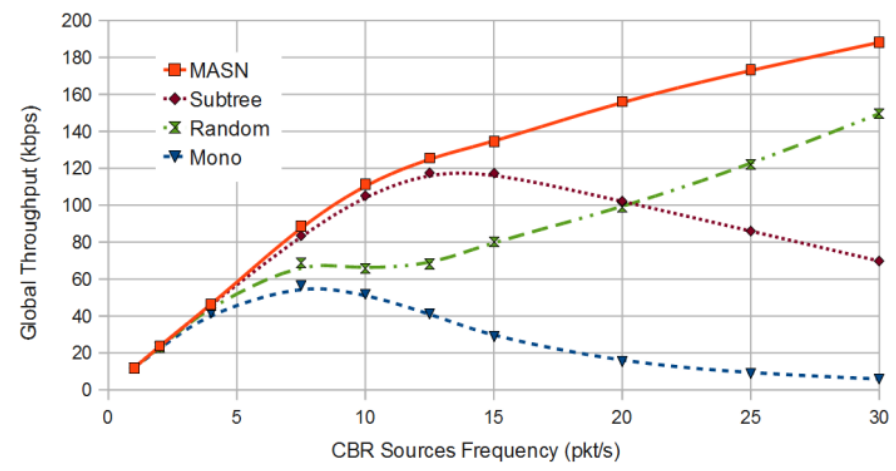

Figure 4. Global Thoughput/CBR Sources Frequency

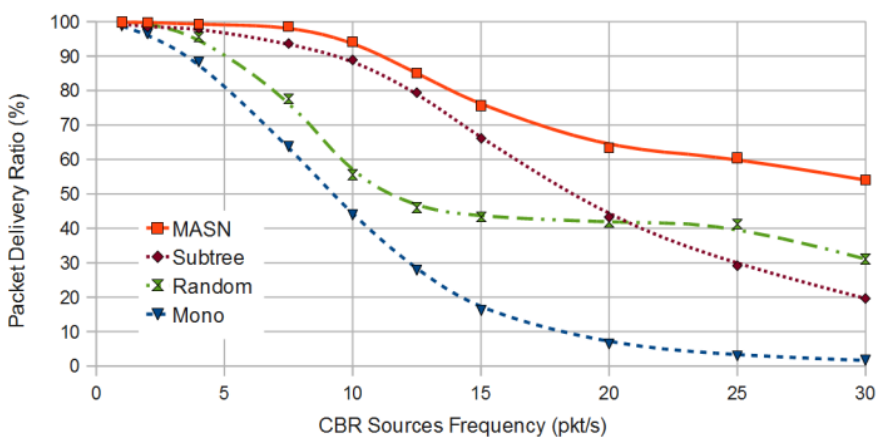

Figure 5. Packet Delivery Ratio/CBR Sources Frequency

Figures 6 and 7 show the same metrics as the number of CBR sources increases. The sources are gradually activated on the different subtrees (one source per subtree, then two sources per subtree ...) to delay the contentions, particularly in the mono-channel solution. In this case, the difference in performance between the solutions is less important, in particular for the Subtree solution (the progressive activation of the CBR sources favors this solution) but MASN protocol gives a much higher throughput for a delivered packets ratio close to $90 \%$.

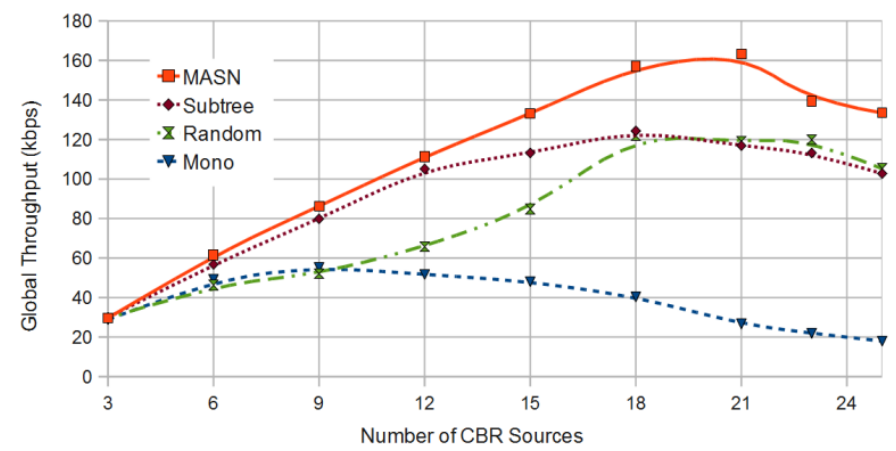

Figure 6. Global Thoughput/Number of CBR Sources

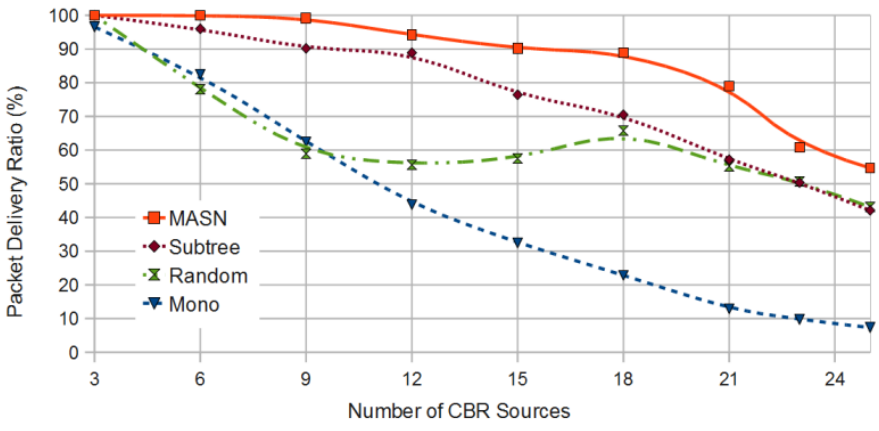

Figure 7. Packet Delivery Ratio/Number of CBR Sources

\section{CONCLUSION}

We have studied and tested a multichannel allocation solution for hierarchical ZigBee/IEEE 802.15.4 networks corresponding to realistic situations (less than 50 nodes with a hierarchical routing and many-to-one transmissions) with as main objective the bandwidth improvement. The principal interest of this solution resides in its simplicity and the facility to integrate it in IEEE 802.15.4 devices insofar as the modifications of the MAC layer are light. This solution is efficient: according to the intensity of the traffic, the global throughput is multiplied by a variable factor between 2 and 5 in comparison with the mono-channel solution and with packets loss ratio much smaller. The throughput is also improved by an average factor equal to 2 compared to a random channel allocation or to a simple subtree distribution.

We currently work on several improvements. The first consists in allocating in a centralized way several channels on each link, the selection of the channel being done dynamically starting from the LQI or the RSSI available on CC2420. The second idea consists in improving the CC2420 transceiver packet forwarding process in the case of multihop links.

Besides, the following stage is the implementation and the test of the multichannel solution on our real sensor network platform made up of MicaZ, TelosB and Imotes2.

\section{REFERENCES}

[1] Zigbee, "Zigbee Specification," ZigBee Standards Organization, Standard Zigbee 053474r17, January 2008

[2] IEEE 802.15, "Part 15.4: Wireless medium access control (MAC) and physical layer (PHY) specifications for low-rate wireless personal area networks (WPANs)," ANSI/IEEE, Standard 802.15.4 R2006, 2006.

[3] Datasheet for Chipcon/Texas Instruments CC2420 $2.4 \mathrm{GHz}$ IEEE 802.15.4/ZigBee-Ready RF Tranceiver, http://focus.ti.com/lit/ds/symlink/cc2420.pdf [referenced September 2008].

[4] MEMSIC Company http://www.memsic.com/products/wireless-sensornetworks.html

[5] Ian F. Akyildiz , Tommaso Melodia and Kaushik R. Chowdhury, « A survey on wireless multimedia sensor networks », Journal of Computer Networks, Volume 51, Issue 4, 2007

[6] R. Maheshwari, H.Gupta and S. R. Das, "Multichannel MAC Protocols for Wireless Networks", In Proceedings of IEEE SECON 2006, Reston, VA, Sept 2006.

[7] J. So and N. Vaidya,. "Multi-channel mac for ad hoc networks: Handling multi-channel hidden terminals using a single transceiver". In Proc. ACM MobiHoc, 2004. 
[8] J. Zhang, G. Zhou, C. Huang, S. H. Son, and J. A.. Stankovic, "TMMAC: An Energy Efficient Multi-Channel MAC Protocol for Ad Hoc Networks," in Proc. of IEEE ICC, June 2007.

[9] P. Bahl, R. Chandra, and J. Dunagan. "SSCH: Slotted seeded channel hopping for capacity improvement in ieee 802.11 adhoc wireless networks", In Proc. of ACM MobiCom, 2004

[10] M. Jovanovic and G. Djordjevic, "TFMAC: Multi-channel MAC Protocol for Wireless Sensor Networks", in Proc. of 8-th International Conference on Telecommunications in Modern Satellite, Cable and Broadcasting Services (TELSIKS), Nis, Serbia, September 2007.

[11] G. Zhou, C. Huang, T. Yan, T. He, J.A. Stankovic and T.F. Abdelzaher, "MMSN: Multi-Frequency Media Access Control forWireless Sensor Networks," in IEEE INFOCOM2006, 2006.

[12] H. Soroush, M. Salajegheh, A. Kalis, "HyMAC: Hybrid TDMA/FDMA Medium Access Control Protocol for Wireless Sensor Networks", in IEEE PIMRC 2007
[13] Y. Wu, J. Stankovic, and T. He, "Realistic and Efficient Multi-Channel Communications in Wireless Sensor Networks", in IEEE INFOCOM, April 2008

[14] Xun Chen Peng Han Qiu-Sheng He Shi-liang Tu Zhang-Long Chen, "A Multi-Channel MAC Protocol for Wireless Sensor Networks", The Sixth IEEE International Conference on Computer and Information Technology, 2006, Seoul, September 2006, pp: 224-224.

[15] M. Petrova, J. Riihijarvi, P. Mahonen, S. Labella, "Performance study of IEEE 802.15.4 using measurements and simulations, IEEE Wireless Communications and Networking Conference," 2006. WCNC 2006.

[16] The Network Simulator NS-2, http://www.isi.edu/nsnam/ns/

[17] F. Österlind and A. Dunkels, "Approaching the Maximum 802.15.4 Multi-hop Throughput “ In Proceedings of HotEmNets 2008. 